\title{
Haptic Teleoperation under Variable Delay and Actuator Saturation
}

\author{
F. Hashemzadeh ${ }^{\mathrm{a}, \mathrm{b}}$, M. Tavakoli ${ }^{\mathrm{b}}$, I. Hassanzadeh ${ }^{\mathrm{a}, \mathrm{b}}$ \\ ${ }^{a}$ Faculty of Electrical and Computer Engineering, University of Tabriz, Tabriz, Iran \\ ${ }^{\mathrm{b}}$ Department of Electrical and Computer Engineering, University of Alberta, Edmonton, Alberta, Canada \\ E-mails: \{hashemzadeh, izadeh\}@tabrizu.ac.ir and \{farzad.hashemzadeh, mahdi.tavakoli, ihassanz\}@ualberta.ca
}

\begin{abstract}
In this paper, a novel control scheme is proposed to guarantee global asymptotic stability of bilateral teleoperation systems that are subject to time-varying time delays in their communication channel and "sandwich linearity" in their actuators. This extends prior art concerning control of nonlinear bilateral teleoperation systems under time-varying time delays to the case where the local and the remote robots' control signals pass through saturation or similar nonlinearities that belong to a class of systems we name sandwich linear systems. We call the proposed method nonlinear proportional plus damping $(n P+D)$. The asymptotic stability of the closed-loop system is established using a Lyapunov-Krasovskii functional under conditions on the controller parameters, the actuator saturation characteristics, and the maximum values of the time-varying time delays. The controller is experimentally validated on a pair of 3-DOF PHANToM Premium 1.5A robots, which have limited actuation capacity, that form a teleoperation system with a varying-delay communication channel.
\end{abstract}

Keywords - Nonlinear teleoperation, varying time delay, sandwich linearity, actuator saturation, asymptotic stability.

\section{INTRODUCTION}

In telerobotic applications with a distance between the local and the remote robots, there will be a time delay in the communication channel of the system, which can destabilize it [1]. In practice, the communication delay can be time-varying and asymmetric in the forward and backward paths between the operator and the remote environment. On the other hand, in any practical teleoperation system, the actuator output (i.e., control signal) has a limited amplitude, i.e., is subject to saturation. Controllers that ignore actuator saturation may cause undesirable responses and even closed-loop instability [2]. Although it may be possible to avoid actuator saturation by using sufficiently high-torque actuators in robots, the large size of the actuators will cause further problems in robot design and control. Therefore, it is desirable to develop control methods that take any actuator saturation into account at the design outset and, therefore, allow for efficient and stable control with small-size actuators that inevitably possess a limited output capacity. In order to address the stability of position control for a single robotic manipulator subjected to bounded actuator output, several approaches have been proposed in the literature. An anti-windup approach is presented to guarantee global asymptotic stability of Euler-Lagrange systems in [3]. In [4], a controller is proposed involving a gravity compensation term plus a saturating function through which the position errors pass. A velocity and position feedback method with adaptive gravity compensation is reported in [5] in which the velocity and position errors separately pass through two nonlinear saturating functions and the outputs are then added to an adaptive gravity compensation term. In [6], a brief review of PD plus gravity compensation controllers are provided. None of the above has been done in the context of teleoperation systems.

Recently, there has been some attention paid to actuator saturation in bilateral teleoperation systems. Combining wave variables with a nonlinear proportional controller, an architecture to handle actuator saturation is discussed in [7] assuming the delay IEEE World Haptics Conference 2013

14-18 April, Daejeon, Korea

978-1-4799-0088-6/13/\$31.00 @2013 IEEE in the communication channel is constant. In [8], an anti-windup approach combined with wave variables is used for constant-delay teleoperation subjected to bounded control signals.

In this paper, a control scheme is introduced to cope with actuator saturation in nonlinear bilateral teleoperation systems that are subjected to time-varying delays. Asymptotic stability of the position error in the teleoperation system is studied, resulting in conditions on the controller parameters, the actuator saturation characteristics and the maximum values of varying delays. This paper is organized as follows. Section II states the preliminaries while the proposed control and main results are presented in Sections III and IV respectively. In Section V, experimental results are provided followed by conclusions in Section VI.

Notation. We denote the set of real numbers by $R=(-\infty, \infty)$, the set of positive real numbers by $R_{>0}=(0, \infty)$, and the set of nonnegative real numbers by $R_{\geq 0}=[0, \infty)$. Also, $\|X\|_{\infty}$ and $\|X\|_{2}$ stand for the Euclidian $\infty$-norm and 2-norm of a vector $X \epsilon R^{n \times 1}$, and $|X|$ denotes element-wise absolute value of the vector $X$. The $\mathcal{L}_{\infty}$ and $\mathcal{L}_{2}$ norms of a time function $f: R_{\geq 0} \rightarrow R^{n \times 1}$ are shown as $\|f\|_{\mathcal{L} \infty}=\sup _{t \in[0, \infty)}\|f(t)\|_{\infty}$ and $\|f\|_{\mathcal{L} 2}=\left(\int_{0}^{\infty}\|f(t)\|_{2}{ }^{2} d t\right)^{1 / 2}$, respectively. The $\mathcal{L}_{\infty}$ and $\mathcal{L}_{2}$ spaces are defined as the sets $\left\{f: R_{\geq 0} \rightarrow R^{n \times 1},\|f\|_{\mathcal{L} \infty}<+\infty\right\}$ and $\left\{f: R_{\geq 0} \rightarrow R^{n \times 1},\|f\|_{\mathcal{L} 2}<\right.$ $+\infty\}$, respectively. For simplicity, we refer to $\|f\|_{\mathcal{L}_{\infty}}$ as $\|f\|_{\infty}$ and to $\|f\|_{\mathcal{L} 2}$ as $\|f\|_{2}$. We also simplify the notation $\lim _{t \rightarrow \infty} f(t)=0$ to $f(t) \rightarrow 0$.

\section{Preliminaries}

\section{A. Model of Teleoperation System}

Consider the master (local) and slave (remote) robots with saturated inputs as follows:

$$
\begin{aligned}
& M_{m}\left(q_{m}(t)\right) \ddot{q}_{m}(t)+C_{m}\left(q_{m}(t), \dot{q}_{m}(t)\right) \dot{q}_{m}+G_{m}\left(q_{m}(t)\right)= \\
& \tau_{h}(t)-S\left(\tau_{m}(t)\right) \\
& M_{s}\left(q_{s}(t)\right) \ddot{q}_{s}(t)+C_{s}\left(q_{s}(t), \dot{q}_{s}(t)\right) \dot{q}_{s}(t)+G_{s}\left(q_{s}(t)\right)= \\
& S\left(\tau_{s}(t)\right)-\tau_{e}(t)
\end{aligned}
$$

Here, $q_{i}, \dot{q}_{i}$ and $\ddot{q}_{i} \in R^{n \times 1}$ for $i \epsilon\{m, s\}$ are the joint positions, velocities and accelerations of the master and slave robots, respectively. Also, $M_{i}\left(q_{i}(t)\right) \in R^{n \times n}, C_{i}\left(q_{i}(t), \dot{q}_{i}(t)\right) \in R^{n \times n}$, and $g\left(q_{i}(t)\right) \in R^{n \times 1}$ are the inertia matrix, the Coriolis/centrifugal matrix, and the gravitational vector, respectively. Moreover, $\tau_{h}$ and $\tau_{e} \epsilon R^{n \times 1}$ are torques applied by the human operator and the environment, respectively. Lastly, $\tau_{m}$ and $\tau_{s} \in R^{n \times 1}$ are the control torques for the master and the slave robots. Note that the control torques are skewed by the vector function $S: R^{n \times 1} \rightarrow R^{n \times 1}$, which can be nonlinear.

Important properties of the nonlinear dynamic model (1)-(2) are $([9,10])$

P-1. For a manipulator with revolute joints, the inertia matrix $M(q)$ is symmetric positive-definite and has the following upper and lower bounds:

$0<\lambda_{\min }(M(q(t))) I \leq M(q(t)) \leq \lambda_{M a x}(M(q(t))) I \leq \infty$ where $I \epsilon R^{n \times n}$ is the identity matrix.

P-2. For a manipulator, the relation between the Coriolis/centrifugal and the inertia matrices is as follows: $\dot{M}(q(t))=C(q(t), \dot{q}(t))+C^{T}(q(t), q(t))$ 
P-3. For a manipulator with revolute joints, there exists a positive $\eta$ bounding the Coriolis/centrifugal term as $\|C(q(t), x(t)) y(t)\|_{2} \leq \eta\|x(t)\|_{2}\|y(t)\|_{2}$

P-4. The time derivative of $C(q(t), \dot{q}(t))$ is bounded if $\dot{q}(t)$ and $\ddot{q}(t)$ are bounded.

P-5. For a manipulators with revolute joints, the gravity vector $G(q(t))$ is bounded (i.e., there exist positive constants $\gamma_{j}$ such that every element of the gravity vector, $g_{j}(q(t))$, $j=1, \cdots, n$, satisfies $\left.\left|g_{j}(q(t))\right| \leq \gamma_{j}\right)$.

\section{B. Model of Actuator with Sandwich Linearity}

In the following, sandwich linearity of the actuator as a vector function $S(\cdot)$ is introduced. It is assumed that $n$ is the number of joints in the master and slave robots and the elements of $S(X)$, where $X \triangleq\left[x_{1} \cdots x_{n}\right]^{T}$, are $s_{j}\left(x_{j}\right): R \rightarrow R, j=1, \cdots, n$, defined by

$$
s_{j}\left(x_{j}\right)\left\{\begin{array}{ccc}
>M_{j}, & \text { if } & x_{j}>M_{j} \\
=x_{j}, & \text { if } & -M_{j} \leq x_{j} \leq M_{j} M_{j}>0 \\
<-M_{j}, & \text { if } & x_{j}<-M_{j}
\end{array}\right.
$$

With this definition of $S(X)$, it is possible to define different sandwich linearity characterizations for different joints of the manipulator. Note that the function $s_{j}\left(x_{j}\right)$ is only required to be linear for $-M_{j} \leq x_{j} \leq M_{j}$, and can be nonlinear (unbounded or bounded) for $\left|x_{j}\right|>M_{j}$. A well-known example of such a function is saturation.

It is imperative to have $\gamma_{j}<M_{j}, j=1, \cdots, n$, where $\gamma_{j}$ is the upper bound of $\left|g_{j}(q(t))\right|$. This condition implies that the actuators of each of the master and the slave manipulators have the capacity to overcome the corresponding robot's gravity within their workspaces.

\section{Proposed Control Law}

In this paper, a nonlinear Proportional plus Damping $(\mathrm{nP}+\mathrm{D})$ controller that incorporates gravity compensation is proposed for the master and the slave robots:

$\tau_{m}(t)=-G_{m}\left(q_{m}(t)\right)+P\left(q_{m}(t)-q_{s}\left(t-T_{2}(t)\right)\right)+K_{m} \dot{q}_{m}(t)$

$\tau_{s}(t)=G_{s}\left(q_{s}(t)\right)-P\left(q_{s}(t)-q_{m}\left(t-T_{1}(t)\right)\right)-K_{s} \dot{q}_{s}(t)$

Here, $P(X): R^{n \times 1} \rightarrow R^{n \times 1}$ is a vector with elements $p_{j}\left(x_{j}\right): R \rightarrow$ $R$. The function $p_{j}\left(x_{j}\right)$ is required to be strictly increasing, bounded, continuous, passing through the origin, concave for positive $x$ and convex for negative $x$, with continuous first derivative around the origin, such that $\left|p_{j}\left(x_{j}\right)\right| \leq\left|x_{j}\right|$ and $p_{j}\left(-x_{j}\right)=-p_{j}\left(x_{j}\right)$.

Under the above assumptions, we will have the following properties for $p_{j}\left(x_{j}\right)$ :

P-I. For any $x, y \in R,\left|p_{j}(x)-p_{j}(y)\right| \leq 2 p_{j}(|x-y|)$

P-II. For any $x, y \in R$, if $x<y$ then $p_{j}(x)<p_{j}(y)$

P-III. For any $x, y \in R_{\geq 0}, p_{j}(x+y) \leq p_{j}(x)+p_{j}(y)$

P-IV. For any $x \in R, \lim _{\varepsilon \rightarrow 0} p_{j}(\varepsilon x)=\lim _{\varepsilon \rightarrow 0} \varepsilon p_{j}(x)$

P-V. For any $x, y \in R, x p_{j}(y) \leq x p_{j}(x)+y p_{j}(y)$

P-VI. For any $x \in R,\left|p_{j}(x)\right| \leq \min \left\{|x|, N_{j}\right\}$ where $N_{j} \triangleq$ $\sup _{x \in R} p_{j}(x)$

P-VII. For any $x(t) \epsilon R$, time derivative of $p_{j}(x(t))$ is bounded.

P-VIII. For any $x \in R, x p_{j}(x) \geq 0$

\section{Main Results}

Let us start by a few preliminary lemmas that will be needed in the proof of our first main result in Theorem I. For simplicity, we use $G_{m}(\cdot), G_{s}(\cdot), P_{m}(\cdot)$ and $P_{s}(\cdot)$ instead of $G_{m}\left(q_{m}(t)\right), G_{s}\left(q_{s}(t)\right)$, $P\left(q_{m}(t)-q_{s}\left(t-T_{2}(t)\right)\right) \quad$ and $P\left(q_{s}(t)-q_{m}\left(t-T_{1}(t)\right)\right)$, respectively. Similarly, we denote by $g_{m_{j}}(\cdot), g_{s_{j}}(\cdot), p_{m_{j}}(\cdot)$ and $p_{s_{j}}(\cdot)$ the $j$-th element of $G_{m}(\cdot), G_{s}(\cdot), P_{m}(\cdot)$ and $P_{s}(\cdot)$, respectively.

Lemma I. Given $g_{m_{j}}(\cdot) \leq \gamma_{j}<M_{j}, g_{s_{j}}(\cdot) \leq \gamma_{j}<M_{j}, p_{m_{j}}(\cdot) \leq$ $N_{j} \leq M_{j}-\gamma_{i}$ and $p_{s_{j}}(\cdot) \leq N_{j} \leq M_{j}-\gamma_{i}$, for positive-definite diagonal matrices $K_{m}$ and $K_{s}$ the following inequalities hold:
$\dot{q}_{m}^{T}\left(S\left(G_{m}(\cdot)-P_{m}(\cdot)-K_{m} \dot{q}_{m}\right)-\left(G_{m}(\cdot)-P_{m}(\cdot)\right)\right) \leq 0$

$\dot{q}_{s}^{T}\left(S\left(G_{s}(\cdot)-P_{s}(\cdot)-K_{s} \dot{q}_{s}\right)-\left(G_{s}(\cdot)-P_{s}(\cdot)\right)\right) \leq 0$

Lemma II. For any $T(t) \in R_{\geq 0}, x(t) \in R_{\geq 0}$, we have

$p_{i}\left(\int_{t-T(t)}^{t} x(\tau) d \tau\right) \leq \int_{t-T(t)}^{t} p_{i}(x(\tau)) d \tau$

Lemma III. For any vector functions $q_{m}(t) \triangleq\left[q_{m_{1}}(t) \cdots q_{m_{n}}(t)\right]^{T} \quad$ and $\quad q_{s}(t) \triangleq\left[q_{s_{1}}(t) \cdots q_{s_{n}}(t)\right]^{T}$ and for any positive time-varying scalars $T_{1}(t)$ and $T_{2}(t)$, the following inequality holds:

$\dot{q}_{m}^{T}(t)\left(P\left(q_{m}(t)-q_{s}(t)\right)-P\left(q_{m}(t)-q_{s}\left(t-T_{2}(t)\right)\right)\right) \leq$

$2\left|\dot{q}_{m}(t)\right|^{T} \int_{t-T_{2}(t)}^{t} P\left(\left|\dot{q}_{s}(\tau)\right|\right) d \tau$

$\dot{q}_{s}^{T}(t)\left(P\left(q_{s}(t)-q_{m}(t)\right)-P\left(q_{s}(t)-q_{m}\left(t-T_{1}(t)\right)\right)\right) \leq$

$2\left|\dot{q}_{s}(t)\right|^{T} \int_{t-T_{1}(t)}^{t} P\left(\left|\dot{q}_{m}(\tau)\right|\right) d \tau$

where $\dot{q}_{m}(t)$ and $\dot{q}_{s}(t)$ are the time derivatives of $q_{m}(t)$ and $q_{s}(t)$, respectively.

Lemma IV. For any vectors $A(t) \triangleq\left[a_{1}(t) \cdots a_{n}(t)\right]^{T}$ and $B(t) \triangleq\left[b_{1}(t) \cdots b_{n}(t)\right]^{T}$ and for any bounded time-varying scalar $0 \leq T(t) \leq T_{m}$, the following inequality holds:

$A^{T}(t) \int_{t-T(t)}^{t} P(B(\tau)) d \tau-\int_{t-T(t)}^{t} B^{T}(\tau) P(B(\tau)) d \tau \leq T_{m} A^{T}(t) P(A(t))$

Proofs of the above four lemmas are provided in Appendix.

Theorem I. Assuming the human operator and the environment are passive, in the bilateral teleoperation system (1)-(2) with controllers (4)-(5), the velocities $\dot{q}_{m}$ and $\dot{q}_{s}$ and the position error $q_{m}-q_{s}$ are bounded for any bounded time-varying time delays $T_{1}(t)$ and $T_{2}(t)$ provided that $T_{1 \max }+T_{2 \max } \leq \frac{-d+M_{\min }}{2 N_{\max }}, 2\left(T_{1 \max }+\right.$ $\left.T_{2 \max }\right)<K_{s}, 2\left(T_{1 \max }+T_{2 \max }\right)<K_{m}$ and $\gamma_{j}+N_{j} \leq M_{j}$.

In the above $M_{\text {min }} \triangleq \min \left\{M_{j}\right\}$ and $N_{\text {max }} \triangleq \max \left\{N_{j}\right\}$ for $j=1 \cdots n$. Also, $d_{m} \triangleq \sup _{(x, y) \in R^{n \times 1} \times R^{n \times 1} \| G_{m}}(x)-P(y) \|_{\infty}$,

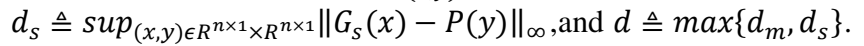
Lastly, $T_{1 \max } \triangleq \sup _{t \in R} T_{1}(t)$ and $T_{2 \max } \triangleq \sup _{t \in R} T_{2}(t)$.

Proof of Theorem I: Applying controller (4)-(5) to the system (1)(2), we have following closed loop dynamics:

$M_{m}\left(q_{m}(t)\right) \ddot{q}_{m}(t)+C_{m}\left(q_{m}(t), \dot{q}_{m}(t)\right) \dot{q}_{m}(t)+G_{m}\left(q_{m}(t)\right)=$

$-S\left(-G_{m}\left(q_{m}(t)\right)+P\left(q_{m}(t)-q_{s}\left(t-T_{2}(t)\right)\right)+K_{m} \dot{q}_{m}(t)\right)+\tau_{h}(t)$

$M_{s}\left(q_{s}(t)\right) \ddot{q}_{s}(t)+C_{s}\left(q_{s}(t), \dot{q}_{s}(t)\right) \dot{q}_{s}(t)+G_{s}\left(q_{s}(t)\right)=$

$+S\left(G_{s}\left(q_{s}(t)\right)-P\left(q_{s}(t)-q_{m}\left(t-T_{1}(t)\right)\right)-K_{s} \dot{q}_{s}(t)\right)-\tau_{e}(t)$

To show the stability of the system (10)-(11), take

$V(t)=V_{1}(t)+V_{2}(t)+V_{3}(t)+V_{4}(t)$

$V_{1}(t)=\frac{1}{2} \dot{q}_{m}^{T}(t) M_{m}\left(q_{m}(t)\right) \dot{q}_{m}(t)$

$+\frac{1}{2} \dot{q}_{s}^{T}(t) M_{s}\left(q_{s}(t)\right) \dot{q}_{s}(t)$

$V_{2}(t)=\int_{0}^{t}\left(-\dot{q}_{m}(\tau) \tau_{h}(\tau)+\dot{q}_{s}(\tau) \tau_{e}(\tau)\right) d \tau$

$V_{3}(t)=\sum_{j=1}^{n} \int_{0}^{q_{m_{j}}(t)-q_{s_{j}}(t)} p_{j}\left(\gamma_{j}\right) d \gamma_{j}$

$V_{4}(t)=2 \int_{-T_{1 \max }}^{0} \int_{t+\gamma}^{t} \dot{q}_{m}^{T}(\eta) P\left(\dot{q}_{m}(\eta)\right) d \eta d \gamma$

$+2 \int_{-T_{2 \max }}^{0} \int_{t+\gamma}^{t} \dot{q}_{s}^{T}(\eta) P\left(\dot{q}_{s}(\eta)\right) d \eta d \gamma$

Note that, based on the assumption of passivity of the operator and the environment, $V_{2}(t)$ is a lower-bonded function. In other words, there exist positive constants $\kappa_{m}$ and $\kappa_{s}$ such that

$\int_{0}^{t}\left(-\dot{q}_{m}(\tau) \tau_{h}(\tau)\right) d \tau+\kappa_{m} \geq 0 \quad$ and $\quad \int_{0}^{t}\left(\dot{q}_{s}(\tau) \tau_{e}(\tau)\right) d \tau+\kappa_{s} \geq 0$

Considering property $\mathrm{P}-2$, the time derivative of $V_{1}(t)$ becomes 
$\dot{V}_{1}(t)=\dot{q}_{m}^{T}(t)\left(-S\left(P\left(q_{m}(t)-q_{s}\left(t-T_{2}(t)\right)\right)-G_{m}\left(q_{m}(t)\right)+\right.\right.$

$\left.\left.K_{m} \dot{q}_{m}(t)\right)\right)+\dot{q}_{m}^{T}(t)\left(\tau_{h}(t)-G_{m}\left(q_{m}(t)\right)\right)$

$+\dot{q}_{s}^{T}(t)\left(S\left(G_{s}\left(q_{s}(t)\right)-P\left(q_{s}(t)-q_{m}\left(t-T_{1}(t)\right)\right)-\right.\right.$

$\left.\left.K_{s} \dot{q}_{s}(t)\right)\right)+\dot{q}_{s}^{T}(t)\left(-\tau_{e}(t)-G_{s}\left(q_{s}(t)\right)\right)$

The time derivatives of $V_{2}(t)$ and $V_{3}(t)$ are

$\dot{V}_{2}(t)=\dot{q}_{m}(t) \tau_{h}(t)-\dot{q}_{s}(t) \tau_{e}(t)$

$\dot{V}_{3}(t)=\left(\dot{q}_{m}(t)-\dot{q}_{s}(t)\right) P\left(q_{m}(t)-q_{s}(t)\right)$

By adding and subtracting each of $\dot{q}_{m}(t) P\left(q_{m}(t)-\right.$ $\left.q_{s}\left(t-T_{2}(t)\right)\right)$ and $\dot{q}_{s}(t) P\left(q_{s}(t)-q_{m}\left(t-T_{1}(t)\right)\right)$ to and from $V_{1}$ and noting that $(-X)=-P(X)$, it is possible to see that $\dot{V}_{1}(t)+\dot{V}_{2}(t)+\dot{V}_{3}(t)=$

$\dot{q}_{m}^{T}(t)\left\{S\left(G_{m}\left(q_{m}(t)\right)-P\left(q_{m}(t)-q_{s}\left(t-T_{2}(t)\right)\right)-K_{m} \dot{q}_{m}(t)\right)\right.$

$\left.-\left(G_{m}\left(q_{m}(t)\right)-P\left(q_{m}(t)-q_{s}\left(t-T_{2}(t)\right)\right)\right)\right\}$

$+\dot{q}_{m}^{T}(t)\left\{P\left(q_{m}(t)-q_{s}(t)\right)-P\left(q_{m}(t)-q_{s}\left(t-T_{2}(t)\right)\right)\right\}$

$+\dot{q}_{s}^{T}(t)\left\{s\left(G_{s}\left(q_{s}(t)\right)-P\left(q_{s}(t)-q_{m}\left(t-T_{1}(t)\right)\right)-K_{s} \dot{q}_{s}(t)\right)\right.$

$\left.-\left(G_{s}\left(q_{s}(t)\right)-P\left(q_{s}(t)-q_{m}\left(t-T_{1}(t)\right)\right)\right)\right\}$

$+\dot{q}_{s}^{T}(t)\left\{P\left(q_{s}(t)-q_{m}(t)\right)-P\left(q_{s}(t)-q_{m}\left(t-T_{1}(t)\right)\right)\right\}$

Considering Lemma I, it is easy to see that there exist positive $\delta_{m}$ and $\delta_{s}$ such that

$\dot{V}_{1}(t)+\dot{V}_{2}(t)+\dot{V}_{3}(t) \leq-\delta_{m} K_{m} \dot{q}_{m}^{T}(t) \dot{q}_{m}(t)-\delta_{s} K_{s} \dot{q}_{s}^{T}(t) \dot{q}_{s}(t)$

$+\dot{q}_{m}^{T}(t)\left\{P\left(q_{m}(t)-q_{s}(t)\right)-P\left(q_{m}(t)-q_{s}\left(t-T_{2}(t)\right)\right)\right\}$

$+\dot{q}_{s}^{T}(t)\left\{P\left(q_{s}(t)-q_{m}(t)\right)-P\left(q_{s}(t)-q_{m}\left(t-T_{1}(t)\right)\right)\right\}$

where $\delta_{m}$ and $\delta_{s}$ are defined as

$\delta_{m} \triangleq \frac{-1}{K_{m}\left\|\dot{q}_{m}(t)\right\|_{2}^{2}} \dot{q}_{m}^{T}(t)\left\{S\left(G_{m}\left(q_{m}(t)\right)-P\left(q_{m}(t)-q_{s}\left(t-T_{2}(t)\right)\right)\right.\right.$

$\left.\left.-K_{m} \dot{q}_{m}(t)\right)-\left(G_{m}\left(q_{m}(t)\right)-P\left(q_{m}(t)-q_{s}\left(t-T_{2}(t)\right)\right)\right)\right\},\left\|\dot{q}_{m}(t)\right\|_{2} \neq 0$

$\delta_{s} \triangleq \frac{-1}{K_{s}\left\|\dot{q}_{s}(t)\right\|_{2}^{2}} \dot{q}_{s}^{T}(t)\left\{S\left(G_{s}\left(q_{s}(t)\right)-P\left(q_{s}(t)-q_{m}\left(t-T_{1}(t)\right)\right)\right.\right.$

$\left.\left.-K_{s} \dot{q}_{s}(t)\right)-\left(G_{s}\left(q_{s}(t)\right)-P\left(q_{s}(t)-q_{m}\left(t-T_{1}(t)\right)\right)\right)\right\},\left\|\dot{q}_{s}(t)\right\|_{2} \neq 0$

(22)

Applying the result of Lemma III to the last two terms in the right hand side of (21), we get

$\dot{V}_{1}(t)+\dot{V}_{2}(t)+\dot{V}_{3}(t) \leq-\delta_{m} K_{m} \dot{q}_{m}^{T}(t) \dot{q}_{m}(t)-\delta_{s} K_{s} \dot{q}_{s}^{T}(t) \dot{q}_{s}(t)$

$+2\left|\dot{q}_{m}(t)\right|^{T} \int_{t-T_{2}(t)}^{t} P\left(\left|\dot{q}_{s}(\tau)\right|\right) d \tau$

$+2\left|\dot{q}_{s}(t)\right|^{T} \int_{t-T_{1}(t)}^{t} P\left(\left|\dot{q}_{m}(\tau)\right|\right) d \tau$

On the other hand, the time derivative of $V_{4}(t)$ is

$$
\begin{gathered}
\dot{V}_{4}(t)=2 T_{1 \max } \dot{q}_{m}^{T}(t) P\left(\dot{q}_{m}(t)\right)-2 \int_{t-T_{1}(t)}^{t} \dot{q}_{m}^{T}(\tau) P\left(\dot{q}_{m}(\tau)\right) d \tau \\
+2 T_{2 \max } \dot{q}_{s}^{T}(t) P\left(\dot{q}_{s}(t)\right)-2 \int^{t^{2}} \dot{q}_{s}^{T}(\tau) P\left(\dot{q}_{s}(\tau)\right) d \tau
\end{gathered}
$$

Considering (23) and (24) together, we have

$\dot{V}(t) \leq-\delta_{m} K_{m} \dot{q}_{m}^{T}(t) \dot{q}_{m}(t)+2\left|\dot{q}_{m}(t)\right|^{T} \int_{t-T_{2}(t)} P\left(\left|\dot{q}_{s}(\tau)\right|\right) d \tau$

$-\delta_{s} K_{s} \dot{q}_{s}^{T}(t) \dot{q}_{s}(t)+2\left|\dot{q}_{s}(t)\right|^{T} \int_{t-T_{1}(t)}^{t} P\left(\left|\dot{q}_{m}(\tau)\right|\right) d \tau$

$+2 T_{1 \max } \dot{q}_{m}^{T}(t) P\left(\dot{q}_{m}(t)\right)-2 \int_{t-T_{1}(t)}^{t} \dot{q}_{m}(\tau) P\left(\dot{q}_{m}(\tau)\right) d \tau$

$+2 T_{2 \max } \dot{q}_{s}^{T}(t) P\left(\dot{q}_{s}(t)\right)-2 \int_{t-T_{2}(t)}^{t} \dot{q}_{s}^{T}(\tau) P\left(\dot{q}_{s}(t)\right) d \tau$

Applying Lemma IV to (25), we get

$\dot{V}(t) \leq-\delta_{m} K_{m} \dot{q}_{m}^{T}(t) \dot{q}_{m}(t)-\delta_{s} K_{s} \dot{q}_{s}^{T}(t) \dot{q}_{s}(t)+2\left(T_{1 \max }+\right.$

$\left.T_{2 \max }\right) \dot{q}_{m}^{T}(t) P\left(\dot{q}_{m}(t)\right)+2\left(T_{1 \max }+T_{2 \max }\right) \dot{q}_{s}^{T}(t) P\left(\dot{q}_{s}(t)\right)$

Defining $\eta_{m}$ and $\eta_{s}$ as

$\eta_{m}=\frac{\dot{q}_{m}^{T}(t) P\left(\dot{q}_{m}(t)\right)}{\left\|\dot{q}_{m}(t)\right\|_{2}^{2}}, \quad \quad\left\|\dot{q}_{m}(t)\right\|_{2} \neq 0$

$\eta_{s}=\frac{\dot{q}_{s}^{T}(t) P\left(\dot{q}_{s}(t)\right)}{\left\|\dot{q}_{s}(t)\right\|_{2}^{2}}, \quad \quad\left\|\dot{q}_{s}(t)\right\|_{2} \neq 0$ we have

$\dot{V}(t) \leq-\left(\delta_{m} K_{m}-2\left(T_{1 \max }+T_{2 \max }\right) \eta_{m}\right) \dot{q}_{m}^{T}(t) \dot{q}_{m}(t)$

$-\left(\delta_{s} K_{s}-2\left(T_{1 \max }+T_{2 \max }\right) \eta_{s}\right) \dot{q}_{s}^{T}(t) \dot{q}_{s}(t)$

Now, let us find conditions on $\delta_{m}$ and $\delta_{s}$ such that $\dot{V}(t) \leq 0$. It is possible to see from (29) that a sufficient condition for $\dot{V}(t) \leq 0$ is $\delta_{m} K_{m} \geq 2\left(T_{1 \max }+T_{2 \max }\right) \eta_{m}$

$\delta_{s} K_{s} \geq 2\left(T_{1 \max }+T_{2 \max }\right) \eta_{s}$

(31)

Next, we will investigate conditions under which the inequalities (30) and (31) are satisfied. Given the definitions of $\eta_{m}$ and $\eta_{s}$ in (27) and (28) and using property P-VI, we have

$\eta_{m} \leq \min \left\{1, \frac{\sum_{j=1}^{n}\left|\dot{q}_{m_{j}}(t)\right| N_{j}}{\left\|\dot{q}_{m}(t)\right\|_{2}^{2}}\right\}$

$\eta_{s} \leq \min \left\{1, \frac{\sum_{j=1}^{n}\left|\dot{q}_{s_{j}}(t)\right| N_{j}}{\left\|\dot{q}_{s}(t)\right\|_{2}^{2}}\right\}$

To study the lower bounds of $\delta_{m}$ and $\delta_{s}$ for replacement in (30)(31), let us consider two regions $X_{1}$ and $X_{2}$ as

$x_{1} \triangleq\left\{\dot{q}_{m}(t):\left|\dot{q}_{m_{j}}(t)\right| \leq \frac{M_{j}-d_{j}}{K_{m}} j=1 \cdots n\right\}$

$x_{2} \triangleq\left\{\dot{q}_{m}(t):\left|\dot{q}_{m_{j}}(t)\right|>\frac{M_{j}-d_{j}}{K_{m}} j=1 \cdots n\right\}$

where $d_{j} \triangleq \sup _{(x, y) \in R \times R}\left|g_{j}(x)-p_{j}(y)\right|$. We distinguish the following two cases:

- Case 1: $\dot{q}_{m}(t) \in \mathcal{X}_{1}$

Based on $g_{m_{j}}()-.p_{j}()-.K_{m} \dot{q}_{m_{j}}(t) \leq g_{m_{j}}()-.p_{j}()+$. $K_{m}\left|\dot{q}_{m_{j}}(t)\right| \leq g_{m_{j}}()-.p_{j}()+.M_{j}-d_{j} \leq M_{j}$ and the definition of $\delta_{m}$ in (22), we have $\delta_{m}=1$. Also, from (32), we know that $\eta_{m} \leq 1$.

Applying $\delta_{m}=1$ and $\eta_{m} \leq 1$ to (30), the following inequality is found as a sufficient condition for (30):

$K_{m}>2\left(T_{1 \max }+T_{2 \max }\right)$

Therefore, (35) is a sufficient condition to have $-\left(\delta_{m} K_{m}-\right.$ $\left.2\left(T_{1 \max }+T_{2 \max }\right) \eta_{m}\right) \dot{q}_{m}^{T}(t) \dot{q}_{m}(t) \leq 0$.

- Case 2: $\dot{q}_{m}(t) \epsilon \mathcal{X}_{2}$

Then, $\left|g_{m_{j}}()-.p_{j}()-.K_{m} \dot{q}_{m_{j}}(t)\right|$ could be greater than $M_{j}$ or less than $M_{j}$. So,

1. If $\left|g_{m_{j}}()-.p_{j}()-.K_{m} \dot{q}_{m_{j}}(t)\right| \leq M_{j}$, based on the definition of $\delta_{m}$ in (22), $\delta_{m}=1$.

2. If $\left|g_{m_{j}}()-.p_{j}()-.K_{m} \dot{q}_{m_{j}}(t)\right|>M_{j} \quad$, then $s_{i}\left(g_{m_{j}}()-\right.$. $\left.p_{j}()-.K_{m} \dot{q}_{m_{j}}(t)\right)>M_{j}$ and using reverse triangular inequality, we will have the following inequality:

$$
\begin{array}{r}
\left|\left(g_{m_{j}}(.)-p_{j}(.)\right)-\left(s_{i}\left(g_{m_{j}}(.)-p_{j}(.)-K_{m} \dot{q}_{m_{j}}(t)\right)\right)\right| \\
>\left|M_{j}-\right| g_{m_{j}}(.)-p_{j}(.)||>M_{j}-d_{j}
\end{array}
$$

Using Lemma I, we have

$$
\begin{aligned}
& \dot{q}_{m_{j}}(t)\left(\left(g_{m_{j}}(.)-p_{j}(.)\right)-\left(s_{j}\left(g_{m_{j}}(.)-p_{j}(.)-K_{m} \dot{q}_{m_{j}}(t)\right)\right)\right)= \\
& \left|\dot{q}_{m_{j}}(t)\right|\left|\left(g_{m_{j}}(.)-p_{j}(.)\right)-\left(s_{j}\left(g_{m_{j}}(.)-p_{j}(.)-K_{m} \dot{q}_{m_{j}}(t)\right)\right)\right|
\end{aligned}
$$

Given that $M_{j}-d_{j} \geq M_{\min }-d$ and based on the definition of $\delta_{m}$ in (22), we get

$\delta_{m}>\frac{\sum_{i=1}^{n}\left|\dot{q}_{m_{j}}(t)\right|\left(M_{\min }-d\right)}{K_{m}\left\|\dot{q}_{m}(t)\right\|_{2}^{2}}$

Knowing from (32) that $\eta_{m} \leq\left(\sum_{j=1}^{n}\left|\dot{q}_{m_{j}}(t)\right| N_{j}\right) /\left(\left\|\dot{q}_{m}(t)\right\|_{2}^{2}\right)$ and using (37), it is possible to see that $\delta_{m} K_{m} N_{\text {max }} \geq\left(M_{\text {min }}-d\right) \eta_{m}$.

Using this, we can find the following condition to satisfy the inequality (30): 
$\frac{M_{\min }-d}{N_{\max }} \geq 2\left(T_{1 \max }+T_{2 \max }\right)$

Therefore, if (35) and (38) are satisfied, then $-\left(\delta_{m} K_{m}-\right.$ $\left.2\left(T_{1 \max }+T_{2 \max }\right) \eta_{m}\right) \dot{q}_{m}^{T}(t) \dot{q}_{m}(t) \leq 0$. Finally, conducting a similar analysis to find a condition for (31) to hold will result in (38) and the following inequality:

$K_{s}>2\left(T_{1 \max }+T_{2 \max }\right)$

Using the above analysis, it is possible to see that if $M_{\max }$ satisfies (38) and $K_{m}$ and $K_{s}$ fulfill inequalities (35) and (39), then $\dot{V}(t) \leq 0$ meaning that all terms in $V(t)$ are bounded. Thus, $\dot{q}_{m}, \dot{q}_{s}$ and $q_{m}-q_{s} \in \mathcal{L}_{\infty}$ and the poof is complete.

Note that the parameter $d$ defined in Theorem $\mathrm{I}$ is equal to $N_{\text {max }}+\gamma_{\text {max }}$ where $\gamma_{\max } \triangleq \max \left\{\gamma_{j}\right\}$. Using the inequalities (35), (38) and (39), a schematic representation of the stability condition in terms of $T_{1 \max }$ and $T_{2 \max }$ is shown in Figure. 1.

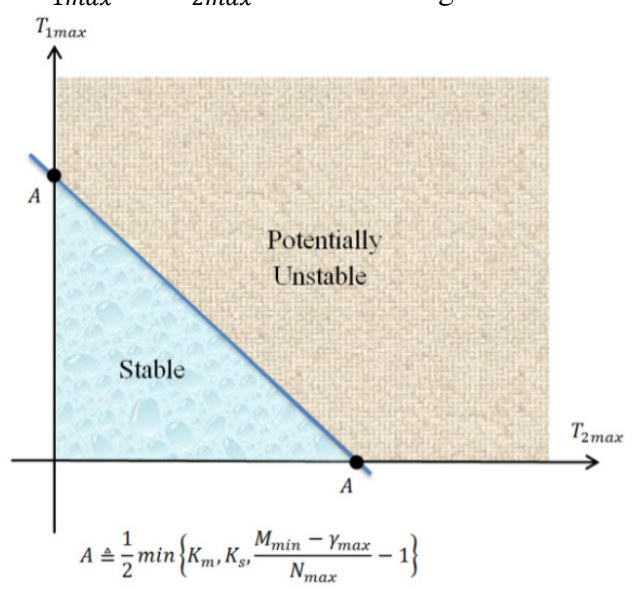

Figure 1a. The stability region determined by inequalities (35), (38) and (39).

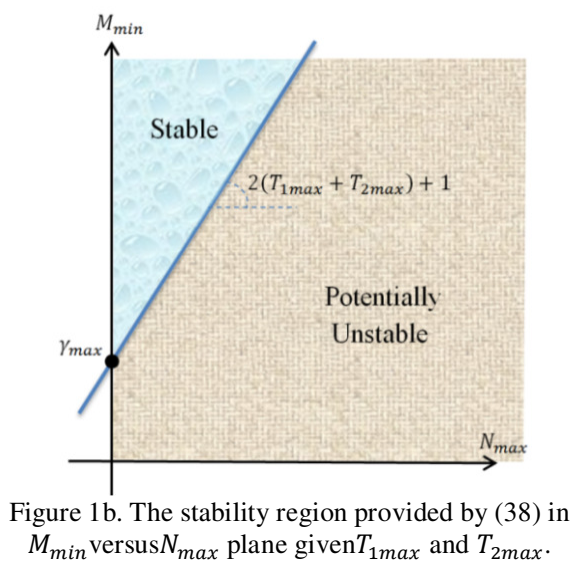

Theorem II. In the bilateral teleoperation system (1)-(2) with the controller (4)-(5), the absolute values of the velocities $\left|\dot{q}_{m}(t)\right|$ and $\left|\dot{q}_{s}(t)\right|$ and the position error $\left|q_{m}(t)-q_{s}(t)\right|$ tend to zero asymptotically in free motion (i.e., $\tau_{h}(t), \tau_{e}(t) \rightarrow 0$ ) if all conditions in Theorem I are satisfied and both $\dot{T}_{1}(t)$ and $\dot{T}_{2}(t)$ are bounded.

\section{Proof of Theorem II:}

Integrating both sides of (29), it is possible to see that $\dot{q}_{m}(t)$ and $\dot{q}_{s}(t) \in \mathcal{L}_{2}$. Based on the result of Theorem I, $V(t)$ is a lower bounded decreasing function. Therefore, $\dot{q}_{m}(t), \dot{q}_{s}(t)$, and $q_{m}(t)-$ $q_{s}(t) \epsilon \mathcal{L}_{\infty}$. Using the fact that $q_{m}(t)-q_{s}\left(t-T_{2}(t)\right)=q_{m}(t)-$ $q_{s}(t)+\int_{t-T_{2}(t)}^{t} \dot{q}_{s}(t) d t$ and $\int_{t-T_{2}(t)}^{t} \dot{q}_{s}(t) d t \in \mathcal{L}_{\infty}$, we have $q_{m}(t)-$ $q_{s}\left(t-T_{2}(t)\right) \epsilon \mathcal{L}_{\infty}$. Since the gravity terms $g_{m}$ and $g_{s}$ are bounded, using property $\mathrm{P}-1$ and $\mathrm{P}-2$ of system dynamics and given the boundedness of $S\left(\tau_{m}(t)\right)$ and $S\left(\tau_{s}(t)\right)$, it can be seen that $\ddot{q}_{m}(t)$ and $\ddot{q}_{s}(t) \epsilon \mathcal{L}_{\infty}$. Because $\dot{q}_{m}(t) \epsilon \mathcal{L}_{2}$ and $\ddot{q}_{m}(t) \epsilon \mathcal{L}_{\infty}$, using Barbalat's lemma we have that $\dot{q}_{m}(t) \rightarrow 0$. Similarly, it can be reasoned that $\dot{q}_{s}(t) \rightarrow 0$.

Now, if $\ddot{q}_{m}$ and $\ddot{q}_{s}$ are continuous in time, or equivalently $\dddot{q}_{m}(t)$ and $\dddot{q}_{s}(t) \in \mathcal{L}_{\infty}$, then $\dot{q}_{m}(t)$ and $\dot{q}_{s}(t) \rightarrow 0$ ensures that $\ddot{q}_{m}(t)$ and $\ddot{q}_{s}(t) \rightarrow 0$ (due to Barbalat's lemma). Let us investigate the boundedness of $\dddot{q}_{i}(t)$ for $i \epsilon\{m, s\}$. The closed-loop dynamics found from combining the open-loop system (1) and (2) with the controllers (4) and (5) is

$\ddot{q}_{m}(t)=\left(M_{m}\left(q_{m}(t)\right)\right)^{-1}\left\{-C_{m}\left(q_{m}(t), \dot{q}_{m}(t)\right) \dot{q}_{m}(t)-G_{m}\left(q_{m}(t)\right)\right.$

$\left.-S\left(-G_{m}\left(q_{m}(t)\right)+P\left(q_{m}(t)-q_{s}\left(t-T_{2}(t)\right)\right)+K_{m} \dot{q}_{m}(t)\right)\right\}$

$\ddot{q}_{s}(t)=\left(M_{s}\left(q_{s}(t)\right)\right)^{-1}\left\{-C_{s}\left(q_{s}(t), \dot{q}_{s}(t)\right) \dot{q}_{s}(t)-G_{s}\left(q_{s}(t)\right)\right.$

$\left.-S\left(-G_{s}\left(q_{s}(t)\right)+P\left(q_{s}(t)-q_{m}\left(t-T_{1}(t)\right)\right)+K_{s} \dot{q}_{s}(t)\right)\right\}$

Differentiating both sides with respect to time and given

$\frac{d}{d t}\left(M_{i}\left(q_{i}(t)\right)\right)^{-1}=-\left(M_{i}\left(q_{i}(t)\right)\right)^{-1}\left\{C_{i}\left(q_{i}(t), \dot{q}_{i}(t)\right)\right.$

$\left.+C_{i}^{T}\left(q_{i}(t), \dot{q}_{i}(t)\right)\right\} M_{i}\left(q_{i}(t)\right) \quad i \in\{m, s\}$

and based on properties P-1 and P-3 and given the boundedness of $\dot{q}_{s}$ and $\dot{q}_{m}$, it is easy to see that $\frac{d}{d t}\left(M_{m}\left(q_{m}(t)\right)\right)^{-1}$ and $\frac{d}{d t}\left(M_{s}\left(q_{s}(t)\right)\right)^{-1}$ are bounded. Given that $\dot{P}(\cdot)$ is bounded and using properties $\mathrm{P}-1, \mathrm{P}-3$ and $\mathrm{P}-4$ of the system dynamics and the boundedness of $q_{s}(t)-q_{m}\left(t-T_{1}(t)\right), q_{m}(t)-q_{s}\left(t-T_{2}(t)\right)$ $, \dot{q}_{m}, \dot{q}_{s}, \ddot{q}_{m}, \ddot{q}_{s}, \dot{T}_{1}$ and $\dot{T}_{2}$, it can be seen that $\dddot{q}_{m}$ and $\dddot{q}_{s}$ are bounded. Given that $\dot{q}_{m}(t)$ and $\dot{q}_{s}(t) \rightarrow 0$ and $\dddot{q}_{m}(t)$ and $\dddot{q}_{s}(t) \in \mathcal{L}_{\infty}$, using Barbalat's lemma we have that $\ddot{q}_{m}(t)$ and $\ddot{q}_{s}(t) \rightarrow 0$.

Considering the dynamic equations of the master and slave robots in (10) and (11), having shown that $\ddot{q}_{i}(t) \rightarrow 0$ and $\dot{q}_{i}(t) \rightarrow$ $0, i \in\{m, s\}$, it is easy to see that

$G_{m}\left(q_{m}(t)\right) \rightarrow S\left(G_{m}\left(q_{m}(t)\right)-P\left(q_{m}(t)-q_{s}\left(t-T_{2}(t)\right)\right)\right)$

$G_{s}\left(q_{s}(t)\right) \rightarrow S\left(G_{s}\left(q_{s}(t)\right)-P\left(q_{s}(t)-q_{m}\left(t-T_{1}(t)\right)\right)\right)$

Given $S\left(\mathrm{G}_{\mathrm{i}}\left(\mathrm{q}_{\mathrm{i}}(\mathrm{t})\right)\right)=\mathrm{G}_{\mathrm{i}}\left(\mathrm{q}_{\mathrm{i}}(\mathrm{t})\right)$, we find that

$P\left(q_{m}(t)-q_{s}\left(t-T_{2}(t)\right)\right) \rightarrow 0$

$P\left(q_{s}(t)-q_{m}\left(t-T_{1}(t)\right)\right) \rightarrow 0$

and using assumptions on $\mathrm{P}(\cdot)$,

$\left|q_{m}(t)-q_{s}\left(t-T_{2}(t)\right)\right| \rightarrow 0$

$\left|q_{s}(t)-q_{m}\left(t-T_{1}(t)\right)\right| \rightarrow 0$

It is possible to see the asymptotic zero convergence of velocities and the position tracking error and proof is completed.

Remark I. Based on the assumption $T_{1 \max }+T_{2 \max } \leq\left(M_{\min }-\right.$ $\left.\left(N_{\max }+\gamma_{\max }\right)\right) /\left(2 N_{\max }\right)$ in Theorem I, it is possible to see a tradeoff between the robustness to the maximum values of time delays and the tracking performance in controller design. For instance, if $N_{\text {max }}$ is lowered, then the position difference between the master and the slave robots contributes less to the control signal, resulting in an increase in the settling time for the position tracking response. At the same time, as $N_{\max }$ is lowered, the maximum admissible values of time delays increase; i.e., the robustness of the system stability to larger time delays improves. The above trends in performance and stability are understandable once one pays attention to the control laws (4)-(5). When $N_{\max }$ has a small value, the nonlinear proportional terms in (4)-(5) are suppressed, leaving more "room" for the derivative signals $\dot{q}(t)$ to contribute to the control signal, i.e., the velocity gains $K_{m}$ and $K_{s}$ are allowed to be larger. It is clear from the stability conditions (35) and (39) that larger $K_{m}$ and $K_{s}$ allow for larger values for the maximum time delays. At the same time, with a small $N_{\max }$, settling time of position tracking increases, degrading the performance of the teleoperation system. Therefore, it can be concluded that, for a 
fixed $M_{\text {min }}$, there is a trade-off between stability and performance of the system and this trade-off can be tuned by changing $N_{\max }$.

\section{Experimental Results}

To verify the theoretical results of this paper, the master and the slave manipulators are considered to be a pair of 3-DOF PHANToM Premium 1.5A robots that are connected via a communication channel with varying time delays with a uniform distribution between 81 and $100 \mathrm{~ms}$. In the proposed $\mathrm{nP}+\mathrm{D}$ method, the following controllers are used:

$$
\begin{aligned}
& \tau_{m}(t)=-G_{m}\left(q_{m}(t)\right)+0.1 p\left(5\left(q_{m}(t)-q_{s}\left(t-T_{2}(t)\right)\right)\right)+\dot{q}_{m}(t) \\
& \tau_{s}(t)=G_{s}\left(q_{s}(t)\right)-0.1 p\left(5\left(q_{s}(t)-q_{m}\left(t-T_{1}(t)\right)\right)\right)-\dot{q}_{s}(t)
\end{aligned}
$$
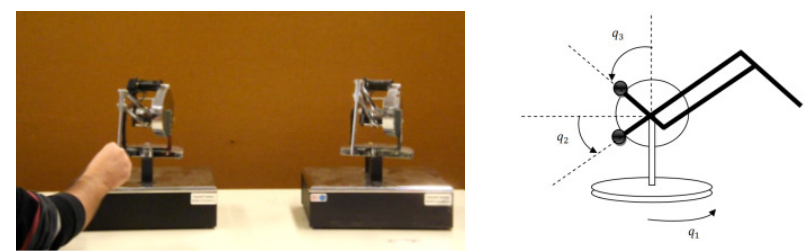

Figure. 2. The experimental teleoperation setup consisting of two PHANToM Premium 1.5A robots and the schematic of the PHANToM robot with its corresponding joint angles.

In the above, we choose the nonlinear function in the controllers to be $p(x) \triangleq \operatorname{sgn}(x) \min \{|x|, 1\}$ with $\operatorname{sgn}(\cdot)$ being the signum function. As a result, when the position errors $q_{m}(t)-$ $q_{s}\left(t-T_{2}(t)\right)$ (the same holds for the position error $q_{s}(t)-$ $q_{m}\left(t-T_{1}(t)\right)$ ) is between -0.2 and 0.2 radian, it appears linearly through a gain of 0.5 in the control signal. Otherwise, its contribution to the control signal is maxed out at -0.1 or 0.1 . Note that the function $0.1 p(5 x)$ meets all the required properties listed in Section III. Also note that the approximate levels of the actuator saturation for the first, second and third joint of the PHANToM robot in generalized coordinate are $M_{1}=0.29, M_{2}=0.29$ and $M_{3}=0.23 \mathrm{Nm}$, respectively. Also $\gamma_{\max }=0.07, M_{\min }=0.28$, $T_{1 \max }=T_{1 \max }=0.1, N_{\max }=0.1$ and it is easy to see that for these values the sufficient condition (38) for stability is satisfied. Experimental results of the proposed $\mathrm{nP}+\mathrm{D}$ controller in terms of joint position tracking between the master and the slave robots are shown in Figure. 3.
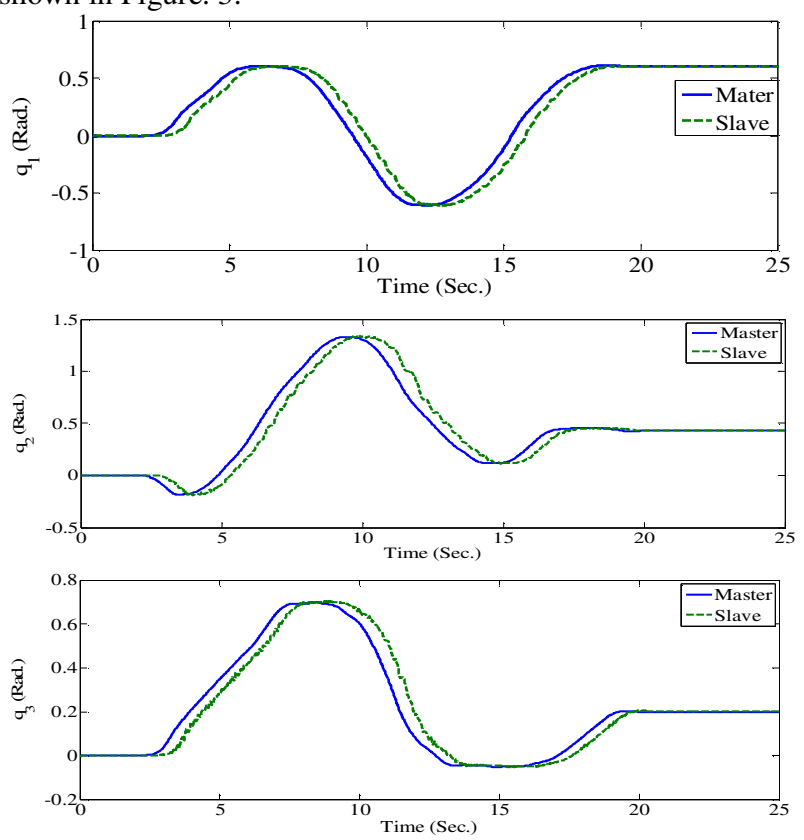

Figure. 3. Joint position tracking between the master and the slave using the proposed $\mathrm{nP}+\mathrm{D}$ control method.

Let us study the effect of $N_{\max }$, which is the maximum value of the nonlinear function $\mathrm{P}(\cdot)$ in (4)-(5), on the performance of the teleoperation system in experiments. To show the slave's joint position trajectory in response to a step set-point (corresponding to a fixed position for the master), the control signal (49) is applied to the slave robot for different values of $N_{\text {max }}$.

$\tau_{s}(t)=G_{s}\left(q_{s}(t)\right)-N_{\max } p\left(5\left(q_{s}(t)-q_{m}\left(t-T_{1}(t)\right)\right)\right)$ where $p($.$) is the same as that used in (48), i.e., p(x) \triangleq$ $\operatorname{sgn}(x) \min \{|x|, 1\}$. In Figure. 4, the step responses of the slave robot's joint positions are shown for three different values of $N_{\text {max }}$. Evidently, a smaller $N_{\text {max }}$, which corresponds to a reduced contribution of the tracking error $q_{s}(t)-q_{m}\left(t-T_{1}(t)\right)$ to the slave robot's control signal, leads to a slower step response (i.e., larger settling time). This is a result that is consistent with Remark I.
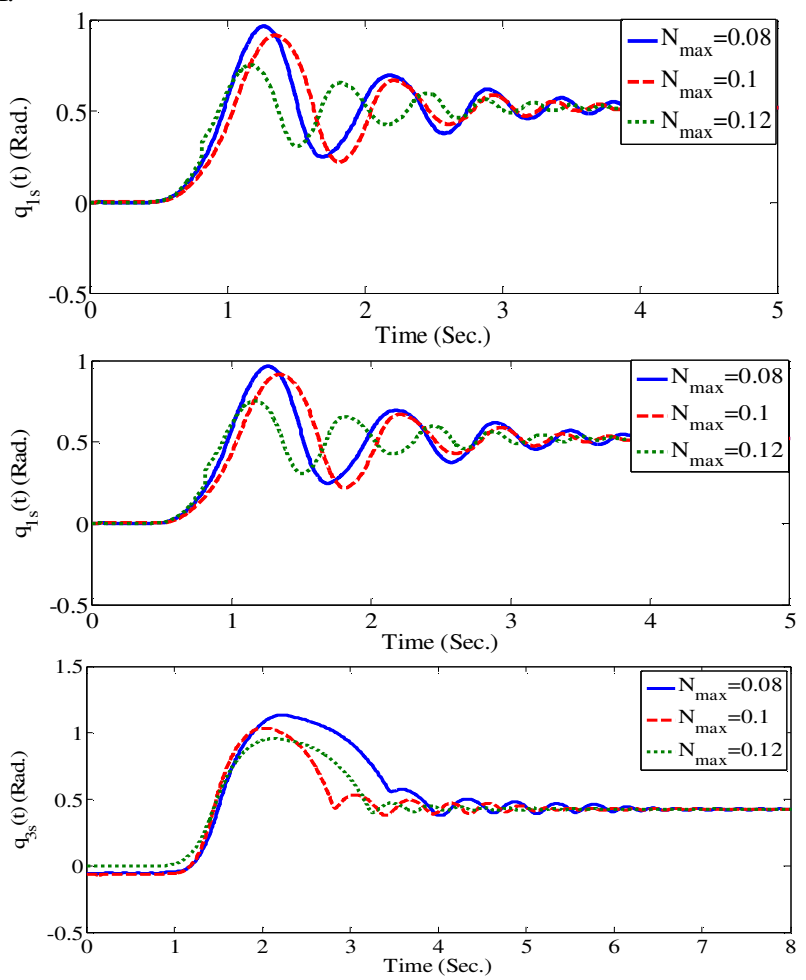

Figure. 4. Step response of slave robot's joint positions for different values of $\mathrm{N}_{\max }$.

\section{Conclusion}

In this paper, we developed a novel method to cope with actuator saturation in bilateral teleoperation systems that are subjected to time-varying time delays in their communication channels. The proposed controller, which we call $\mathrm{nP}+\mathrm{D}$ method, is similar to the conventional $\mathrm{P}+\mathrm{D}$ controller except for the fact that we have replaced the proportional term by a nonlinear function through which the position errors pass. This makes the proposed $\mathrm{nP}+\mathrm{D}$ method capable of handling actuator saturation and guaranteeing position tracking even in the presence of timevarying time delays in the communication channel. We analyzed the stability of the system using a Lyapunov-Krasovskii functional and showed asymptotic position tracking between the master and the slave robots. The derived stability conditions involve relationships between the $\mathrm{nP}+\mathrm{D}$ controller parameters, the actuator saturation characteristics, and the maximum values of the time- 
varying delays. We experimentally tested the proposed controller on a teleoperated pair of 3-DOF PHANToM Premium robots, which are naturally subject to actuator saturation. Simulation and experimental results of the proposed $\mathrm{nP}+\mathrm{D}$ control method have demonstrated its efficiency.

\section{References}

[1] Sheridan, T. B.. Space teleoperation through time delay: Review and prognosis. IEEE Transaction on Robotics, vol. 9, no. 5, pp. 592-606, 1993.

[2] Kothare, M. V., Campo, P. J., Morari, M., Nett, C. N. A unified framework for the study of antiwindup designs. Automatica, vol. 30, no. 12, pp. $1869-1883,1994$.

[3] Morabito, F., Teel, A. R., Zaccarian, L. Nonlinear Antiwindup Applied to Euler-Lagrange Systems. IEEE Transaction on Robotics and Automation, vol. 20, no. 3, pp. 526-573, 2004.

[4] Loria, A., Kelly, R., Ortega, R., Santibañez, V. On global output feedback regulation of Euler-Lagrange systems with bounded inputs. IEEE Transaction on Automatic Control. vol. 42, pp. 1138-1143, 1997.

[5] Zergeroglu, E., Dixon, W., Behal, A., Dawson, D. Adaptive set-point control of robotic manipulators with amplitude-limited control inputs. Robotica, vol. 18, pp. 171-181, 2000.

[6] Zavala-Rio, A., Santibañez, V. Simple extensions of the PD-withgravity-compensation control law for robot manipulators with bounded inputs. IEEE Transaction on Control Systems Technology, vol. 14, no. 5, pp. 958-965, 2006.

[7] Lee, S. J., Ahn, H. S. Synchronization of bilateral teleoperation systems with input saturation. International Conference on Control, Automation and Systems, pp. 1375-1361, 2010.

[8] Lee, S. J., Ahn, H. S. A Study on Bilateral Teleoperation with Input Saturation and Systems. International Conference on Control Automation and Systems, pp.161-166, 2011.

[9] Kelly, R., Santibáñez, V. \& Loria, A. Control of robot manipulators in joint space. Springer., 2005.

[10] Spong, M. W., Hutchinson, S. Vidyasagar, M. Robot modeling and control. Wiley, 2005.

\section{Appendix}

Here, proofs of Lemmas I, II, III and IV are provided.

Proof of Lemma I: The assumption in the lemma can be summed up as $\left|g_{m_{j}}(\cdot)-p_{m_{j}}(\cdot)\right|<M_{j}$ and $\left|g_{s_{j}}(\cdot)-p_{s_{j}}(\cdot)\right|<M_{j}$. Regarding the increasing monotone property of function $s_{j}(\cdot)$, if $\dot{q}_{m_{j}} \geq 0$ then $s_{j}\left(g_{m_{j}}(\cdot\right.$ )$\left.-p_{m_{j}}(\cdot)-k_{j} \dot{q}_{m_{j}}\right) \leq s_{j}\left(g_{m_{j}}(\cdot)-p_{m_{j}}(\cdot)\right)$ and if $\dot{q}_{m_{j}}<0$ then $s_{j}\left(g_{m_{j}}(\cdot\right.$ )$\left.-p_{m_{j}}(\cdot)-k_{j} \dot{q}_{m_{j}}\right)>s_{j}\left(g_{m_{j}}(\cdot)-p_{m_{j}}(\cdot)\right)$. Based on the fact that $g_{m_{j}}(\cdot)-p_{m_{j}}(\cdot)$ belongs to the linear part of $s_{j}, s_{j}\left(g_{m_{j}}(\cdot)-p_{m_{j}}(\cdot)\right)=$ $g_{m_{j}}(\cdot)-p_{m_{j}}(\cdot)$, therefore for all $\dot{q}_{m_{j}}$

$\dot{q}_{m}^{T}\left(S\left(G_{m}(\cdot)-P_{m}(\cdot)-K_{m} \dot{q}_{m}\right)-\left(G_{m}(\cdot)-P_{m}(\cdot)\right)\right) \leq 0$

Similarly $\dot{q}_{s}^{T}\left(S\left(G_{s}(\cdot)-P_{s}(\cdot)-K_{s} \dot{q}_{s}\right)-\left(G_{s}(\cdot)-P_{s}(\cdot)\right)\right) \leq 0$

Proof of Lemma II: Based on the definition of integral, we know that

$$
\begin{aligned}
& \int_{t-T(t)}^{t} x(\tau) d \tau=\lim _{n \rightarrow \infty} \sum_{k=0}^{n-1} \frac{T(t)}{n} x\left(t-T(t)+k \frac{T(t)}{n}\right) \\
& \int_{t-T(t)}^{t} p_{i}(x(\tau)) d \tau=\lim _{n \rightarrow \infty} \sum_{k=0}^{n-1} \frac{T(t)}{n} p_{i}\left(x\left(t-T(t)+k \frac{T(t)}{n}\right)\right)
\end{aligned}
$$

Using the properties P-III and P-IV of $p_{i}($.$) and knowing that x(\tau)$ and $T(t)$ are positive,

$$
\begin{aligned}
& p_{i}\left(\int_{t-T(t)}^{t} x(\tau) d \tau\right)=p_{i}\left(\lim _{n \rightarrow \infty} \sum_{k=0}^{n-1} \frac{T(t)}{n} x\left(t-T(t)+k \frac{T(t)}{n}\right)\right) \\
& \leq \lim _{n \rightarrow \infty} \sum_{k=0}^{n-1} p_{i}\left(\frac{T(t)}{n} x\left(t-T(t)+k \frac{T(t)}{n}\right)\right) \\
& =\lim _{n \rightarrow \infty} \sum_{k=0}^{n-1} \frac{T(t)}{n} p_{i}\left(x\left(t-T(t)+k \frac{T(t)}{n}\right)\right)=\int_{t-T(t)}^{t} p_{i}(x(\tau)) d \tau
\end{aligned}
$$

Proof of lemma III: Considering property P-I of $p_{j}($.$) ,$

$$
\begin{aligned}
& \left|p_{j}\left(q_{m_{j}}(t)-q_{s_{j}}(t)\right)-p_{j}\left(q_{m_{j}}(t)-q_{s_{j}}\left(t-T_{2}(t)\right)\right)\right| \\
& \leq 2 p_{j}\left(\left|q_{s_{j}}(t)-q_{s_{j}}\left(t-T_{2}(t)\right)\right|\right)=2 p_{j}\left(\left|\int_{t-T_{2}(t)}^{t} \dot{q}_{s_{j}}(\tau) d \tau\right|\right) \\
& \text { and similarly } \\
& \quad\left|p_{j}\left(q_{s_{j}}(t)-q_{m_{j}}(t)\right)-p_{j}\left(q_{s_{j}}(t)-q_{m_{j}}\left(t-T_{1}(t)\right)\right)\right| \\
& \quad \leq 2 p_{j}\left(\left|\int_{t-T_{1}(t)}^{t} \dot{q}_{m_{j}}(\tau) d \tau\right|\right)
\end{aligned}
$$

Using property P-II of $p_{j}($.$) and knowing that for any \dot{q} \in R$ and for any positive $T(t),\left|\int_{t-T(t)}^{t} \dot{q}(\tau) d \tau\right| \leq \int_{t-T(t)}^{t}|\dot{q}(\tau)| d \tau$, then

$p_{j}\left(\left|\int_{t-T(t)}^{t} \dot{q}(\tau) d \tau\right|\right) \leq p_{j}\left(\int_{t-T(t)}^{t}|\dot{q}(\tau)| d \tau\right)$

Considering Lemma II,

$p_{j}\left(\int_{t-T(t)}^{t}|\dot{q}(\tau)| d \tau\right) \leq \int_{t-T(t)}^{t} p_{j}(|\dot{q}(\tau)|) d \tau$

$$
\text { Considering (B7), (B9) and (B10), }
$$

$$
\begin{aligned}
\mid p_{j}\left(q_{m_{j}}(t)-q_{s_{j}}(t)\right) & -p_{j}\left(q_{m_{j}}(t)-q_{s_{j}}\left(t-T_{2}(t)\right)\right) \mid \\
& \leq 2 \int_{t-T(t)}^{t} p_{j}(|\dot{q}(\tau)|) d \tau
\end{aligned}
$$

and therefore

$\dot{q}_{m}^{T}(t)\left(P\left(q_{m}(t)-q_{s}(t)\right)-P\left(q_{m}(t)-q_{s}\left(t-T_{2}(t)\right)\right)\right)$

$=\sum_{j=1}^{n}\left(\dot{q}_{m_{j}}(t)\left(p_{j}\left(q_{m_{j}}(t)-q_{s_{j}}(t)\right)-p_{j}\left(q_{m_{j}}(t)-q_{s_{j}}\left(t-T_{2}(t)\right)\right)\right)\right)$

$\leq \sum_{j=1}^{n}\left(\left|\dot{q}_{m_{j}}(t)\right|\left|p_{j}\left(q_{m_{j}}(t)-q_{s_{j}}(t)\right)-p_{j}\left(q_{m_{j}}(t)-q_{s_{j}}\left(t-T_{2}(t)\right)\right)\right|\right)$

$=2 \sum_{j=1}^{n}\left(\left|\dot{q}_{m_{j}}(t)\right|\left(\int_{t-T(t)}^{t} p_{j}\left(\left|\dot{q}_{s_{j}}(\tau)\right|\right) d \tau\right)\right)$

$=2\left|\dot{q}_{m}(t)\right|^{T} \int_{t-T_{2}(t)}^{t} P\left(\left|\dot{q}_{s}(\tau)\right|\right) d \tau$

Similarly

$\dot{q}_{s}^{T}(t)\left(P\left(q_{s}(t)-q_{m}(t)\right)-P\left(q_{s}(t)-q_{m}\left(t-T_{1}(t)\right)\right)\right)$

$\leq 2\left|\dot{q}_{s}(t)\right|^{T} \int_{t-T_{1}(t)}^{t} P\left(\left|\dot{q}_{m}(\tau)\right|\right) d \tau$

Proof of Lemma IV: given property $\mathrm{P}-\mathrm{V}$ of function $p_{j}($.$) ,$

$\sum_{j=1}^{n}\left(a_{j}(t) p_{j}\left(b_{j}(\tau)\right)-b_{j}(\tau) p_{j}\left(b_{j}(\tau)\right)\right) \leq \sum_{j=1}^{n}\left(a_{j}(t) p_{j}\left(a_{j}(t)\right)\right)$

Therefore,

$$
A^{T}(t) P(B(\tau))-B^{T}(\tau) P(B(\tau)) \leq A^{T}(t) P(A(t))
$$

Integrating both sides based on $d \tau$ from $t-T(t)$ to $t$,

$$
\begin{aligned}
& \int_{t-T(t)}^{t} A^{T}(t) P(B(\tau)) d \tau-\int_{t-T(t)}^{t} B^{T}(\tau) P(B(\tau)) d \tau \\
& \leq \int_{t-T(t)}^{t} A^{T}(t) P(A(t)) d \tau
\end{aligned}
$$

This can be simplified to

$$
A^{T}(t) \int_{t-T(t)}^{t} P(B(\tau)) d \tau-\int_{t-T(t)}^{t} B^{T}(\tau) P(B(\tau)) d \tau \leq T(t) A^{T}(t) P(A(t))
$$

Given property P-VIII of function $p_{j}(\cdot), A^{T}(t) P(A(t))>0$ and so $A^{T}(t) \int_{t-T(t)}^{t} P(B(\tau)) d \tau-\int_{t-T(t)}^{t} B^{T}(\tau) P(B(\tau)) d \tau \leq T_{m} A^{T}(t) P(A(t))$ 